\title{
2015 ASMS Fall Workshop: Lipids and Lipidomics
}

\author{
Gavin Reid ${ }^{1}$ and Xianlin $\operatorname{Han}^{2}$ \\ ${ }^{1}$ University of Melbourne, Victoria, Australia \\ ${ }^{2}$ Sanford Burnham Prebys Medical Discovery Institute-Lake Nona, Florida, USA
}

Gavin Reid (gavin.reid@unimelb.edu.au), the corresponding author.

Over the past two decades, 'lipidomics', defined as "the large-scale study of pathways and networks of cellular lipids in biological systems", has emerged as an independent discipline at the interface of lipid biology, chemistry and technology. A major goal of lipidomic analysis is to characterize the molecular composition of mammalian, bacterial or plant derived lipids isolated from cells, tissues, organs or body fluids (e.g., serum), and to quantitatively monitor their changes in abundance under different physiological conditions, or in the pathogenesis of disease. The insights obtained from these studies are critical toward the development of an improved understanding of the role that lipids play in the regulation of normal cellular function, including as the main structural component of biological membranes, in energy storage, and signaling, and for subsequent functional assays aimed at determining the role of aberrant lipid metabolism or signaling in a variety of pathological conditions.

In order to meet the needs of novice practitioners within the field, the 2015 ASMS Fall Workshop on lipids and lipidomics was organized by Gavin Reid (University of Melbourne) and Xianlin Han (Sanford Burnham Prebys Medical Discovery Institute), and held on November 5-6 at the Catamaran Resort and Spa in San Diego, CA. Specifically, the workshop covered (1) sample handling and lipid extraction; (2) appropriate use of mass spectrometry technologies for characterization, identification, and quantification of individual lipid species with or without chromatographic separation; (3) nomenclature/annotation of lipids analyzed by MS and bioinformatic data analysis strategies; and (4) applications of lipidomics to address biologically relevant questions.

Throughout the two day workshop, nine experts in the field presented 16 tutorial talks to 150 attendees from academic, government, and industrial sectors, from 11 countries. This was the largest turn-out in the history of the ASMS Fall Workshops. Of these, over 40 attendees were graduate students and postdocs, which also represented the largest number in the history of these workshops, and were in part enabled by generous ASMS travel stipends. The talks were grouped into four thematic half-day sessions: (1) Fundamentals of lipids and lipidomics; (2) Characterization and identification of lipids by MS; (3) Quantification, MS lipid imaging, and data processing; and (4) Applications of lipidomics for biological and biomedical research. Each session featured three to five talks of 35 minute duration, plus 10 minute discussions. The interactive nature of the workshop was greatly aided by a real time discussion forum that enabled attendees to post questions during each presentation. Thanks to Professor Stephen Blanksby (Queensland University of Technology) for setting up and moderating this forum. As noted by one of the attendees, "I really like the ability to type in questions for the speakers to address". 
After brief opening remarks from Professor Reid, the workshop began with an 'Introduction to Lipid Structure, Biochemistry and Biology' by Professor Dennis Vance (University of Alberta), who has been actively conducting lipid research for over 50 years and has made numerous seminal contributions to the field. He briefly introduced the complexity of lipids and the relationship between different lipid classes. He overviewed the structure, biosynthesis, and biochemistry of a few classes of lipids, including cholesterol, fatty acids, glycosphingolipids, and phospholipids, and highlighted some of the pioneers who made substantial contributions to early research on these lipids. He also extensively discussed the evolution of lipid research, ranging from their early isolation, characterization of structure and biosynthesis, identification of enzymes and regulations, and determination of functions with knockout animals, through to the current status of lipidomics by using phosphatidylcholine as an example. The presentation clearly summarized that the development of lipidomics has had a long history, we are all standing on the shoulder of giants, and that the new tools of mass spectrometry are leading us into a new era in lipid research.

Professor Han then gave an 'Overview of Lipidomics Workflows' after briefly introducing the definition of lipidomics and the importance of lipidomics tools in lipid research. He discussed that lipidomic analysis is comprised of four key components. These include (1) sample preparation, which includes appropriate sampling, the appropriate number and amounts of (internal) standards, suitable extraction methodologies, and possible derivatization; (2) MS data acquisition by using a variety of approaches such as LC-MS, shotgun lipidomics, MS imaging, and ion mobility techniques; (3) processing of MS data for characterization, identification, and quantification of lipid classes, subclasses, and individual molecular species; and (4) data interpretation based on metabolic pathways and lipid functions, and by using bioinformatics modeling. This presentation particularly emphasized the importance of data quality control prior to data interpretation, and echoed the comments of Professor Vance with regard to the importance of considering lipid metabolic pathways and functions for data interpretation.

Dr. Gerhard Liebisch (University of Regensburg) then gave a talk entitled 'Nomenclature and Systems of Annotation for MS and MS/MS Lipid Analysis'. He pointed out that although IUPAC has recommended a nomenclature system for different lipid species, this system cannot be generally used to reflect the different structural information obtained from MS analyses, because employing distinct analysis methods and/or instruments leads to various levels of structural information. He then discussed these differences with examples, and recommended some rules to annotate MS data that reflect the virtual structural information provided from the MS analysis. These rules can be found in Dr. Liebisch's paper [1].

The last talk of the morning session was given by Dr. M. Cameron Sullards (Georgia Tech) on the topic of 'Sample Preparation and Internal Standards for Lipidomics'. Dr. Sullards first mentioned the importance of using internal standards for lipidomics and the criteria for their selection, including their similarity to the analytes and the practical requirement for using a limited number of lipid internal standards to cover many individual species. He summarized methods for lipid extraction, emphasized those for specific extraction of eicosanoids and sterols, and further mentioned fractionation of lipid extraction with solid phase columns. He finally discussed the usage of internal standards, lipid extraction, and MS methods for sphingolipid 
analysis, giving several examples from his laboratory.

The afternoon session on Characterization and Identification of Lipids by MS featured Professor William Griffiths (Swansea University), who presented on the topic of 'Chromatographic Separation Strategies for Lipid Analysis'. He first overviewed the available matrices for lipid separation including reversed, normal, and ion-exchange phases used to separate lipids based on the hydrophobicities, head group polarities, and charge propensities of lipids, respectively. He then presented the principles of different separation methods for lipid analysis such as LC (including TLC), HPLC, superfluid chromatography, and GC. He then presented a variety of separation strategies in combination with these available materials and methods for analysis of lipids by 1D, 2D, off-line, and on-line LC-MS. He concluded by stating that the strategies used largely depend on the analytical purpose, and highlighted the analysis of neurosteroids in rat brain as an example [2] for the selection of these strategies.

Professor Blanksby (Queensland University of Technology) focused his talk on 'Structural Elucidation of Lipids by Tandem Mass Spectrometry', by highlighting the importance of structural determination for lipid biology and the applications of tandem mass spectrometry (MS/MS) to address the complexity of lipids in biological systems. He discussed the fundamentals of collision-induced dissociation and the mechanisms of lipid ion dissociation in both positive- and negative-ionization modes. He emphasized that understanding the mechanisms of unimolecular dissociation can assist in characterizing new or unexpected lipids, and presented several examples of lipid structure elucidation to highlight that product ions observed in positive- and negative-ionization modes are often complementary and thus it is useful to determine the fragments in both modes. He also recommended literature for further reading, including a review on mechanistic studies [3].

Professor Han returned to present an alternative approach for identification of the individual species of a lipid class of interest, by 'Targeted Lipid Analysis of Using Triple Quadrupole (QqQ) Instruments'. The principle of this approach is that all species of a particular lipid class are comprised of a few building blocks such as a phosphodiester head group and a small number of fatty acyl chains linked to the glycerol molecule in a phospholipid class, and that all these building blocks can be determined by using MS/MS in either precursor-ion or neutral loss scanning modes. The full MS spectrum in the corresponding mass range and the related building blocks form a 2D mass spectrum. Analysis of this 2D spectrum leads to identification of all species (including isomers) of the class of interest. The differential intensities of fatty acyl building blocks resulting from an individual lipid ion can be used to assess the regioisomers. To those classes of lipids that do not carry the building block for a polar head group, derivatization to tag a polar group can be conducted. Furthermore, with the $2 \mathrm{D}$ mass spectra, quantification can also be readily achieved. Professor Han emphasized that one key point for accurate quantification of lipid species is to avoid their aggregation.

Professor Reid presented another alternative approach for high throughput lipidome analysis, involving 'Lipidome Analysis by High Resolution/Accurate Mass Spectrometry'. He discussed the fundamentals of the instrumentation that can be employed to carry out high resolution/accurate mass measurements, as well as instrumentation that provide ultra-high mass resolving power $(>100,000)$, that is necessary to fully resolve isobaric mass cellular lipids in the 
mass range between 600 and 1000 Da. He presented numerous applications of high resolution/accurate mass spectrometry for analysis of cellular lipidomes, but emphasized that some limitations remain when using high mass accuracy instruments, particularly for the resolution of isomeric mass lipids. He outlined and/or showed many different methods that could be used to resolve these issues, including chromatographic separation, differential lipid extraction, polarity switching, derivatization, and MS/MS analysis.

The last talk of the day was given by Professor Blanksby on the topic of 'Emerging Strategies for Comprehensive Lipid Structural Characterization', including for the assignment of regiospecific isomers and isomers with different locations of double bond(s). He emphasized the importance of comprehensive structural characterization in lipidomics, and summarized conventional approaches used for the separation of these isomers by chromatography. He then extensively discussed emerging strategies including radical-directed dissociation, multi-stage MS/MS analysis, ion mobility technology, and chemical derivatization (e.g., ozonolysis and PatternòBüchi reaction). He concluded that challenges of stereochemistry remain and without new tools to differentiate and quantify changes in lipid isomer abundances one could be blind to significant biological changes that may occur between different samples.

The second day of presentations started with a session on Data Processing, with the first talk on 'Relative and Absolute Quantification' given by Dr. Liebisch. In this talk, the importance of using internal standards for quantification was emphasized, and several studies were highlighted that showed the relationship of multiple factors including polar head group and fatty acyl chain (e.g., chain length and double bonds) identities, concentrations, and solvents, on the instrumental response factors of individual lipid species. Considering the complication of lipid aggregation on quantification, a key factor to be controlled is the concentration. Any quantitative analysis of lipids should be conducted at a concentration at which aggregation does not occur. Another important factor to be considered for quantification is the isotope effects due to the differences of carbon numbers present in different lipid species. He exemplified the internal standards used in his laboratory and the approaches used for quantification of different lipid classes.

Professor Robert Murphy (University of Colorado Denver) then presented on the topic of 'Mass Spectrometry Based Lipid Imaging'. The fundamentals of lipid imaging by MALDI-MS were extensively discussed, along with tissue sample preparation for imaging, methods for matrix deposition, tips on mass spectral acquisition, tools for data processing, biological information obtained from imaging, and the use of MS/MS and high resolution to facilitate high quality imaging. Professor Murphy also overviewed some recent advances, including the DESI-based approach for real time cancer surgery, a technique based on laser-induced post ionization, the use of silver nanoparticle matrix to enhance sensitivity, prior chemical/enzyme treatment of tissue, the application of secondary ion techniques, and quantitation in imaging.

The final talk of the morning session was given by Professor Oliver Fiehn (University of California - Davis), who spoke on the topic 'Database Searching and Data Processing'. Due to the complexity of lipids, different adducts, various analytical methods, and differential fragment information from positive- and negative-ionization modes, MS data processing for lipid analysis is one of the most challenge tasks in "omics" research. Multiple programs/software are available for automatic data analysis. Professor Fiehn proposed a general data processing workflow, and 
summarized the commercially and publicly available tools and libraries for lipid identification, including the in silico-generated LipidBlast library [4] developed in his laboratory.

To conclude the morning session, a 45-minute group discussion on the topic of 'Future Directions and Challenges' was held to stimulate involvement from the attendees, and exchange ideas and views on the still evolving lipidomics field. This discussion opportunity appeared to be well-received; attendee feedback received after the workshop included comments such as "The workshop provided an excellent forum for discussions on the challenges of the field. In my view it was high quality for researchers at all levels of expertise".

The afternoon presentations on Applications were started by Professor Reid, who discussed 'Applications of Lipidomics in Disease Biomarker Discovery'. Lipids as disease biomarkers are not new. Cholesterol for atherosclerosis, triglycerides for heart disease and diabetes, etc. have been recognized as lipid biomarkers for many years. However, Professor Reid emphasized that lipidomics enables the accurate and rapid measure of hundreds of individual lipid species (rather than the total content of a given class), which should have higher potential to discover more specific biomarkers for chronic diseases. After reiterating the steps and considerations in a lipidomics study, he presented an overview of multiple studies for the identification of ethercontaining lipids as biomarkers of cancer malignancy and metastatic progression, plasma lipids for stable and unstable coronary artery disease, and plasma phospholipids for antecedent memory impairment.

Professor Murphy then gave a talk on the topic of 'Bioactive Lipid Mediators', which play important roles as secondary messengers of signal transduction and are generally present in very low abundance. He overviewed the types and structures of the lipid mediators, and the challenges to determine their true levels because of autoxidation in sample preparation and noise artifacts in analysis. He compared the choices of MS techniques and their advantages and limitations. He laid out strategies for their isolation, selection of stable isotope standards (purity), and quantitation by multiple reaction monitoring (MRM) methods. He also emphasized data quality control through "looking at data yourself, not relying on computer-generated values without checking". Finally, he challenged the presence of eicosanoids in circulation.

Professor Griffiths then returned to talk on the topic of 'Sterols and Oxysterols', which are present in extreme complexity due to enzymatic and autoxidative generation, are present in various sources (e.g., plants, fungi, and mammals) and play key roles in serving as ligands to nuclear receptors. Analysis of (oxy)sterols is challenging due to the influence of excess background and ready autoxidation of cholesterol, thermal instability, difficulties in ionization. He discussed three layers of analytical methods, including classical GC-MS, modern LC-MS, and advanced LC-MS with derivatization. He summarized a variety of derivatives that are currently available and concluded that Girard derivatization appears to offer the best sensitivity and richest structural information for analysis of (oxy)sterols.

The last talk of the workshop was given by Dr. Sullards on the topic of 'Sphingolipids in Health and Disease'. Sphingolipids are a group of the most structurally complex and diverse eukaryotic lipids, due to a large number of sphingoid backbones, various amide-linked acyl chains with or without carrying a hydroxyl group at different positions, and complex polar head groups. Almost 
all species are bioactive, and are important nutritional components. Metabolic disorders of the majority of sphingolipid classes are associated with a variety of diseases. For example, numerous lysosomal storage diseases are caused by deficiencies in the activities of enzymes that degrade individual sphingolipid classes. Therefore, it is very important to characterize, identify, and quantify sphingolipid species. Dr. Sullards discussed LC-MS, LC-MS/MS, and imaging methods for analysis of this lipid class.

ASMS solicits participant feedback after the workshop on scientific and organizational aspects of the meeting, as well as to solicit suggestions for improvement and for future workshop topics. The attendees uniformly gave high scores for both scientific and organizational aspects. Some examples of the feedback included "The workshop was incredible. It was great to have met and listened to some of the experts in this area, the topics covered were great and the interactions were really helpful. It was absolutely worth bringing my entire lab to the workshop. My students learned a lot", "The meeting was extremely well organized. The speakers were obviously experts in the field but more importantly they were able to disseminate the right data for both introductory and expert levels, as well as answer questions", "I was very pleased with the young enthusiastic crowd. There were plenty of follow up questions after sessions", "We should have this focused workshop every year", "The use of the online forum was excellent - I attended 3 ASMS workshops so far and this one was the richest in terms of discussions. Well done!!" The involvement and interaction between the attendees indeed added much value to the workshop.

We would like to thank the speakers and the attendees for making the workshop such an interactive event. We also thank Jennifer Watson for her spectacular organizational skills in making the workshop run smoothly for all involved.

\section{References:}

[1] Liebisch, G., Vizcaino, J.A., Kofeler, H., Trotzmuller, M., Griffiths, W.J., Schmitz, G., Spener, F., Wakelam, M.J.: Shorthand notation for lipid structures derived from mass spectrometry. J Lipid Res. 54, 1523-1530 (2013).

[2] Liu, S., Sjovall, J., Griffiths, W.J.: Neurosteroids in rat brain: Extraction, isolation, and analysis by nanoscale liquid chromatography-electrospray mass spectrometry. Anal Chem. 75, 5835-5846 (2003).

[3] Hsu, F.F., Turk, J.: Electrospray ionization with low-energy collisionally activated dissociation tandem mass spectrometry of glycerophospholipids: Mechanisms of fragmentation and structural characterization. J Chromatogr B. 877, 2673-2695 (2009).

[4] Kind, T., Liu, K.H., Lee, do Y., Defelice, B., Meissen, J.K., Fiehn, O.: Lipidblast in silico tandem mass spectrometry database for lipid identification. Nat Methods. 10, 755-758 (2013).

Credit for photo:

Photo courtesy of Sergiu P. Palii (University of Florida) 


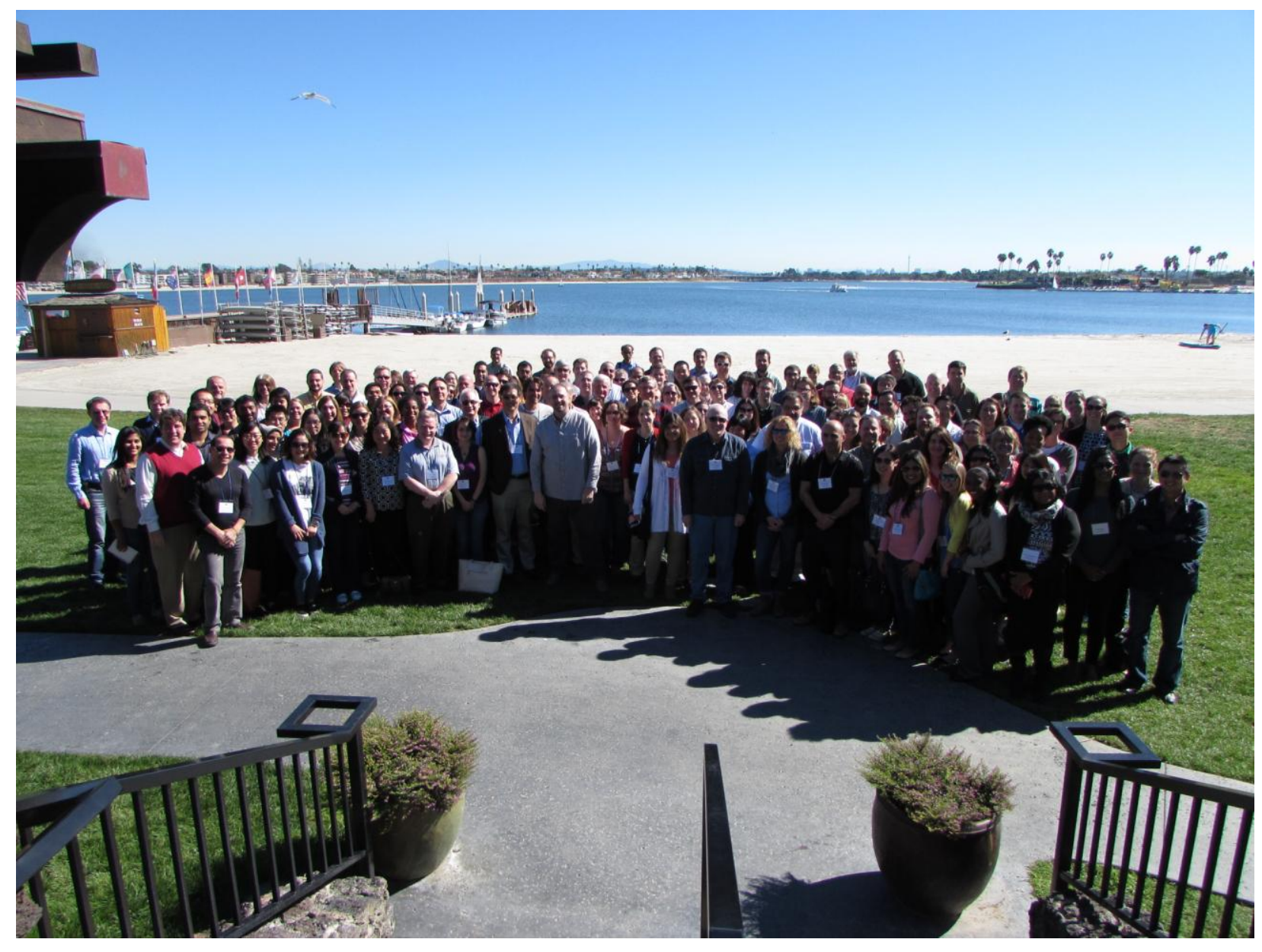




\section{University Library}

\section{- M M N E R VA A gateway to Melbourne's research publications}

Minerva Access is the Institutional Repository of The University of Melbourne

Author/s:

Reid, GE;Han, X

Title:

2015 ASMS Fall Workshop: Lipids and Lipidomics

Date:

2016-05-01

Citation:

Reid, G. E. \& Han, X. (2016). 2015 ASMS Fall Workshop: Lipids and Lipidomics. JOURNAL OF THE AMERICAN SOCIETY FOR MASS SPECTROMETRY, 27, (5), pp.753-756.

SPRINGER. https://doi.org/10.1007/s13361-016-1373-5.

Persistent Link:

http://hdl.handle.net/11343/283327 